\title{
Avaliação do consumo e de metabólitos plasmáticos de cabras gestantes com duas condições corporais alimentadas com dietas formuladas com diferentes níveis de energia
}

\author{
Carla Aparecida Florentino Rodrigues ${ }^{1}$, Marcelo Teixeira Rodrigues ${ }^{2}$, Renata Helena Branco ${ }^{3}$, \\ Giovanni Ribeiro de Carvalho², Robledo de Almeida Torres², Rodolpho de Almeida Torres Filho ${ }^{1}$ \\ 1 Zootecnista. \\ 2 Departamento de Zootecnia da Universidade Federal de Viçosa. \\ ${ }^{3} I Z$ - Sertãozinho.
}

RESUMO - Este estudo foi conduzido com o objetivo de avaliar as relações entre as diferentes condições corporais e os níveis de energia nas dietas sobre o consumo, a variação de peso e a concentração de ácidos graxos não-esterificados (AGNE) no plasma de cabras leiteiras. O período experimental correspondeu aos 30 dias antes da data prevista para o parto. O delineamento experimental foi inteiramente casualizado em esquema fatorial 2 x 3, com duas condições corporais e três níveis de energia líquida (EL) nas dietas. Os animais foram distribuídos em grupos: o valor de escore corporal para o grupo 1 foi menor e, para o grupo 2, maior que 3,25. Foram oferecidas às cabras dietas isonitrogenadas (13\% de PB), com diferentes níveis de EL: 1,$1 ; 1,4$ e 1,6 Mcal/kg de MS. Durante 21 dias antes da data prevista para o parto, foram coletadas amostras de sangue, em intervalos de sete dias, para análises da concentração de AGNE. Analisada individualmente, a condição corporal influenciou os consumos de MS e FDN, de modo que os animais com maior escore $(>3,25)$ apresentaram menor consumo. Os níveis de EL das dietas influenciaram positivamente os consumos de MS e EL no final da gestação e a concentração de AGNE aos 14 dias antes do parto e no dia do parto. As cabras que consumiram a dieta menos energética apresentaram maiores concentrações de AGNE. Portanto, o pico de AGNE durante o final da gestação ocorreu quando o consumo de MS foi reduzido, ou seja, nos últimos 21 dias antes do parto.

Palavras-chave: ácidos graxos não-esterificados, caprinos, consumo, pré-parto, período de transição

\section{Effects of body condition score and dietary energy levels on performance of pregnant dairy goats}

\begin{abstract}
The present study evaluated the relationship between different dietary net energy (NE) levels and body condition score (BCS) on performance of dairy goats during the prepartum period. Forty-eight pregnant goats were kept in individual cages during the last 30 days before parturition. The experimental design was a completely randomized design with a 2 × 3 factorial arrangement of treatments (two BCS and three levels of NE in the diet). Goats were assigned to one of two groups, based on body condition score, which was either 3.25 or below. Prepartum diets contained 1.1, 1.4 or 1.6 Mcal NE/kg DM and 13\% of CP. Blood samples were collected weekly beginning 21 days before parturition and plasma analyzed for concentration of nonesterified fatty acids (NEFA). Intakes of DM and NDF were lower in animals with greater BCS. However, intakes of DM and NE paralleled the increment of energy in the diet while the opposite was observed for NDF intake. As expected, feeding goats the low NE diet increased the plasma concentration of NEFA at day -14 and at parturition.
\end{abstract}

Key Words: goats, intake, nonesterified fatty acids, prepartum, transition period

\section{Introdução}

O período de transição, caracterizado por três semanas anteriores e posteriores ao parto, é marcado pela rápida mudança no metabolismo animal e, particularmente, por uma queda acentuada no consumo de MS, seguida de lenta recuperação no período pós-parto. Nesta fase, a deficiência energética associada ao baixo consumo ocasiona redução na concentração plasmática de glicose e aumento na con- centração de ácidos graxos não-esterificados (AGNE) no plasma sangüíneo, como resultado da mobilização de gordura corporal.

O crescimento fetal durante as últimas semanas de gestação contribui para que a demanda energética seja aumentada. Conseqüentemente, a redução do consumo nesse período pode resultar em balanço energético negativo no animal, alterando os mecanismos de mobilização de energia e contribuindo para um quadro metabólico que 
predispõe o animal a graves problemas nutricionais, como a hipocalcemia, retenção de placenta e toxemia da gestação, além de prejudicar o desempenho produtivo e reprodutivo. Esses distúrbios metabólicos ocorrem com maior freqüência na espécie caprina, em comparação à bovina, visto que a exigência de energia, em relação ao peso do animal, é mais acentuada em caprinos no período pré-parto, especialmente nas cabras com mais de um feto (Moulin, 1991).

A manutenção do balanço energético nas espécies caprina e bovina tem sido alvo de várias pesquisas na última década, em virtude do crescente uso de animais com alto padrão genético e do grande potencial leiteiro. Uma alternativa para amenizar a deficiência energética e a mobilização de gordura das reservas corporais durante o final da gestação é o aumento da densidade energética na dieta (NRC, 1989).

As compilações sobre exigências nutricionais de caprinos têm sido reportadas pelo AFRC (1997) e pelo NRC (1981). Em ambas as publicações, não existem inferências sobre as exigências de cabras durante o período de transição.

O escore da condição corporal, medida subjetiva para avaliar a quantidade de reservas corporais, é determinado pelo acúmulo de gordura no tecido animal e tem sido uma ferramenta freqüentemente utilizada para auxiliar no manejo de rebanho leiteiro. A avaliação do escore de cabras pela condição corporal é baseada na observação tátil, por meio da palpação de áreas específicas, como a região dorsolombar, e da avaliação subjetiva do depósito de tecido adiposo e massa muscular.

Estudos que permitam entender as relações entre diferentes condições corporais e níveis de energia nas dietas durante o final da gestação de cabras leiteiras e seus efeitos sobre os consumos de MS, fibra e energia podem possibilitar o cálculo de dietas balanceadas para caprinos visando máxima eficiência do uso de nutrientes e de energia pelos animais. Portanto, o objetivo neste trabalho foi estudar combinações entre condições corporais e níveis de energia líquida nas dietas sobre o consumo e a concentração de metabólitos plasmáticos de cabras nos últimos 30 dias de gestação (período pré-parto).

\section{Material e Métodos}

A pesquisa foi realizada no Setor de Caprinocultura do Departamento de Zootecnia da Universidade Federal de Viçosa durante o período de maio a julho de 2000.

Foram utilizadas 48 cabras Alpinas, gestantes, mantidas em baias individuais durante os 30 dias finais de gestação.
Os animais foram avaliados quanto ao escore de condição corporal e divididos em dois grupos: o grupo 1 foi composto de cabras com escore inferior a 3,25; e o grupo 2, de animais com escore superior a 3,25, segundo a escala de 1 a 5 proposta por Morand-Fehr e Hervieu (1999), em que 1 corresponde a animal muito magro e 5, a animal obeso. A divisão dos animais em dois grupos de escore de condição corporal (média sugerida de 3,25 ) deveu-se ao fato de este valor representar o ponto médio entre os valores 2,5 e 4,0, considerados limites para classificação de cabras como magras ou gordas, ressaltando-se que o menor escore de condição corporal observado no início do experimento foi 2,0 e o maior, 3,5.

O período pré-experimental de cinco dias foi utilizado com a finalidade de adaptar as cabras às novas dietas e ao período experimental, de 30 dias.

Adotou-se delineamento experimental inteiramente casualizado, em esquema fatorial 2 x 3 (duas condições corporais e três níveis de energia líquida: alto, médio e baixo), com oito repetições.

O nível de energia líquida de 1,4 Mcal/kg MS foi adotado com base nas recomendações do NRC (1981) para cabras no final da gestação, sendo considerado valor padrão de energia ao qual os outros dois níveis, 1,1 (nível baixo) e 1,6 Mcal/kg MS (nível alto), foram comparados (Tabela 1).

Na Tabela 2 constam a descrição dos ingredientes utilizados e a composição química das dietas. As dietas foram formuladas com 13\% de PB (\% MS) para suprirem as exigências nutricionais de proteína, cálcio e fósforo de cabras gestantes (NRC, 1981). O concentrado utilizado foi composto de milho, farelo de soja, uréia, fosfato bicálcico, calcário calcítico e mistura mineral e o volumoso foi feno de capim-tifton (Cynodon spp).

As cabras foram alimentadas duas vezes ao dia (9 e 16h) com dieta na forma de mistura completa, com proporção

Tabela 1 - Condição corporal dos animais e níveis de energia líquida das dietas

Table 1 - Body condition score and levels of net energy of the experimental diets

\begin{tabular}{llc}
\hline $\begin{array}{l}\text { Condição corporal } \\
\text { Body condition score }\end{array}$ & $\begin{array}{c}\text { Nível de energia líquida (Mcal/kg MS) } \\
\text { Net energy (Mcal/kg DM) }\end{array}$ \\
\hline $\begin{array}{l}\text { Grupo 1 (Group 1) } \\
(>3,25)\end{array}$ & Baixo (Low) & 1,1 \\
& Médio (Medium) & 1,4 \\
\hline Grupo 2 (Group 2) & Alto (High) & 1,6 \\
$(<3,25)$ & Baixo (Low) & 1,1 \\
& Médio (Medium) & 1,4 \\
& Alto (High) & 1,6
\end{tabular}


Tabela 2 - Composições percentual (\%MS) e química (\%) das dietas

Table 2 - Ingredient (\%DM) and chemical (\%) composition of the diets

\begin{tabular}{|c|c|c|c|}
\hline & \multicolumn{3}{|c|}{$\begin{array}{c}\text { Nível de energia líquida } \\
\text { (Mcal/kg MS) } \\
\text { Level of net energy (Mcal/kg DM) }\end{array}$} \\
\hline & 1,1 & 1,4 & 1,6 \\
\hline Ingrediente & \multicolumn{3}{|c|}{$\%$ da MS } \\
\hline Ingredient & \multicolumn{3}{|c|}{$\% D M$} \\
\hline Feno de capim-tifton (Tifton grass) & 80,20 & 50,45 & 25,45 \\
\hline Milho moído (Ground corn) & 4,11 & 33,32 & 58,28 \\
\hline Farelo de soja (Soybean meal) & 12,69 & 13,24 & 13,27 \\
\hline Uréia (Urea) & 0,50 & 0,50 & 0,50 \\
\hline Mistura mineral (Mineral mix) & 2,50 & 2,50 & 2,50 \\
\hline \multicolumn{4}{|l|}{$\begin{array}{l}\text { Composição química (\%) } \\
\text { Chemical composition }\end{array}$} \\
\hline $\mathrm{PB}(C P)$ & 13,00 & 13,00 & 13,00 \\
\hline $\mathrm{Ca}$ & 0,75 & 0,75 & 0,75 \\
\hline $\mathrm{P}$ & 0,34 & 0,34 & 0,34 \\
\hline FDN (NDF) & 64,98 & 44,06 & 26,32 \\
\hline FDA $(A D F)$ & 34,76 & 23,10 & 13,22 \\
\hline
\end{tabular}

volumoso:concentrado estabelecida de acordo com o nível de energia líquida da dieta.

A mistura de alimentos oferecida e as sobras foram pesadas diariamente para determinação do consumo diário de MS. A quantidade oferecida foi ajustada considerando a quantidade de sobras observada diariamente (em torno de $10 \%$ da quantidade oferecida) para garantia do consumo voluntário pelos animais. Amostras da dieta oferecida e das sobras foram coletadas três vezes por semana para formação de uma amostra composta. Foram realizadas análises químicas dos componentes da dieta e das sobras para determinação dos teores de MS, FDN, FDA (Van Soest et al., 1991) e PB (Silva, 1990).

As pesagens e as avaliações da condição corporal das cabras foram realizadas de 15 em 15 dias, sempre antes do arraçoamento da manhã, por três dias consecutivos. A avaliação da condição corporal de caprinos se baseou na observação tátil, por meio de palpação da região dorsolombar (Morand-Fehr \& Hervieu, 1999).

Durante os 21 dias antes do parto, foram coletadas amostras de sangue, em intervalos de sete dias, para posteriores análises da concentração de ácidos graxos nãoesterificados (AGNE). O sangue foi coletado da veia jugular em tubos vacutainer heparinizados uma hora e meia após o fornecimento da alimentação da manhã. O plasma sangüíneo foi separado por centrifugação a 3.200 rpm durante 10 minutos e armazenado a $-20^{\circ} \mathrm{C}$. As concentrações plasmáticas de AGNE foram determinadas utilizando-se o kit específico Wako, NEFA C (kit no 994-75409 E), aplicando-se o método enzimático de leitura por colorimetria e densidade ótica com comprimento de onda de $550 \mathrm{~nm}$, modificado por Baumann (1986), citado por Johnson \& Peters (1993).

Na análise estatística, adotou-se o método dos quadrados mínimos, pelo programa SAS (1990), para comparação das médias pelo teste de Student Newman-Keuls (SNK), a $5 \%$ de probabilidade.

\section{Resultados e Discussão}

Não houve interação condição corporal $\times$ nível de energia líquida $(E L)$ nas dietas $(\mathrm{P}>0,05)$ quando avaliados os consumos de MS (CMS), FDN(CFDN) e energia líquida (CEL) dos animais durante os últimos 30 dias de gestação. Do mesmo modo, não houve efeito da interação desses mesmos fatores sobre as concentrações de ácidos graxos não-esterificados (AGNE) durante os últimos 21 dias de gestação.

A análise individual dos fatores comprovou $(\mathrm{P}<0,05)$ efeito da condição corporal sobre o CMS e o CFDN (Tabela 3). Entretanto, a análise individual da condição corporal não comprovou efeito $(\mathrm{P}>0,05)$ desse fator sobre a concentração de AGNE (mM).

Os animais com CC $>3,25$ apresentaram menor consumo de MS e de FDN $(\mathrm{P}<0,05)$ quando comparados às cabras com $C C<3,25$. É provável que as cabras com maior condição corporal, por possuírem maior quantidade de reservas de energia, tiveram suas necessidades nutricionais atendidas com menor quantidade de alimentos, enquanto as mais magras consumiram mais alimento na tentativa de suprir as exigências nutricionais dessa fase e repor ou aumentar alguma reserva de energia.

Como demonstrado na Tabela 4, os níveis de EL nas dietas influenciaram $(\mathrm{P}<0,05)$ os consumos de MS (g/dia; g/kg ${ }^{0,75}$; \%PV), FDN (g/dia e \%PV) e EL (em Mcal/dia e $\mathrm{kcal} / \mathrm{UTM})$.

O aumento do nível de EL nas dietas durante os últimos 30 dias de gestação resultou em incremento no consumo de MS e, conseqüentemente, no consumo de EL. As cabras alimentadas com as dietas com maiores níveis de EL (1,4 e 1,6 Mcal EL/kg MS) apresentaram maior consumo de MS e EL $(P<0,05)$ em comparação àquelas alimentadas com dietas contendo 1,1 Mcal EL/kg MS.

O consumo de FDN foi maior $(\mathrm{P}<0,05)$ entre as cabras alimentadas com a dieta com 1,1 Mcal EL/kg MS (0,90\%PV). Os animais que receberam a dieta com 1,4 Mcal EL/kg MS apresentaram valor intermediário de consumo $(0,67 \% \mathrm{PV})$, 
Tabela 3 - Consumo médio de MS, FDN e energia líquida (EL) em cabras com duas condições corporais durante os últimos 30 dias de gestação

Table 3 - $\quad$ Average intake of DM, NDF, and net energy (NE) on goats with different body condition score during the last 30 days of pregnancy

\begin{tabular}{|c|c|c|}
\hline \multirow[t]{2}{*}{$\begin{array}{l}\text { Consumo } \\
\text { Intake }\end{array}$} & \multicolumn{2}{|c|}{$\begin{array}{l}\text { Condição corporal (CC) } \\
\text { Body condition score (BCS) }\end{array}$} \\
\hline & $\begin{array}{c}\text { Grupo } 1(>3,25) \\
\text { Group } 1(>3.25)\end{array}$ & $\begin{aligned} \text { Grupo } & 2(<3,25) \\
\text { Group } 2 & (<3.25)\end{aligned}$ \\
\hline $\begin{array}{l}\text { MS (g/dia) } \\
D M \text { (g/day) }\end{array}$ & $985,36^{b}$ & $1113,58^{a}$ \\
\hline $\begin{array}{l}\text { FDN (g/dia) } \\
N D F \text { (g/day) }\end{array}$ & $383,51^{b}$ & $462,21^{\mathrm{a}}$ \\
\hline $\begin{array}{l}\text { FDN (\%PV) } \\
\text { NDF (\%BW) }\end{array}$ & $0,61^{\mathrm{b}}$ & $0,69^{a}$ \\
\hline $\begin{array}{l}\text { EL (Mcal/dia) } \\
N E \text { (Mcal/day) }\end{array}$ & 1,40 & 1,55 \\
\hline
\end{tabular}

Médias seguidas de uma mesma letra na linha não diferem pelo teste Student-Newman-Keuls a $5 \%$ de probabilidade.

Means in the same row with different superscripts differ by Student Newman Keuls test at $5 \%$ of probability.

porém, esse valor foi superior ao obtido naquelas alimentadas com dieta formulada com 1,6 Mcal EL/kg MS (0,39\%PV).

É provável que as cabras que consumiram a dieta contendo 1,1 Mcal EL/kg MS e 64,98\% de FDN tenham apresentado limitação do consumo em decorrência da repleção ruminal ocasionada pelo excesso de fibra presente na dieta, visto que o CMS desses animais foi menor que o daqueles que consumiram dietas com 1,4 e 1,6 Mcal EL/kg MS com 44,06 e 26,32\% de FDN, respectivamente. Por outro lado, ao consumirem as dietas com 1,4 e 1,6 Mcal EL/kg MS, os animais podem ter apresentado limitação no consumo de MS e EL, em virtude da ação de mecanismos de controle fisiológico, em comparação àqueles que consumiram a dieta com 1,1 Mcal EL/kg MS. Assim, é possível que fatores nãocontrolados nesse experimento, como palatabilidade da ração, tenham atuado como estimuladores de consumo. Ressalta-se ainda que o material forrageiro era de qualidade mediana a baixa, com alto valor de FDN.

A análise dos consumos de FDN e EL (Figura 1) pode explicar a tendência observada para o consumo de MS das cabras deste experimento. O sistema de predição de consumo em relação à FDN/energia da dieta para as formulações de dietas de ruminantes baseia-se no conceito de que dois mecanismos regulam o consumo dos animais (Mertens, 1985). Segundo Forbes $(1977,1993)$ e Mertens (1987), esses mecanismos de controle fisiológico ou físico podem atuar como limitantes primários de consumo, em virtude da densidade energética e dos teores de fibra das dietas, representando, assim, um diferencial para o entendimento da teoria de controle de consumo em ruminantes. Desse modo, os animais que receberam a dieta com 1,1 Mcal EL/kg
Tabela 4 - Consumos médios de MS, FDN e energia líquida (EL) de cabras alimentadas com dietas formuladas com três níveis de energia durante os últimos 30 dias de gestação

Table 4 - $\quad$ Average intakes of DM (DMI), NDF (NDFI), and net energy (NEI) on goats fed diets with different levels of net energy during the last 30 days of pregnancy

\begin{tabular}{|c|c|c|c|}
\hline \multirow[t]{2}{*}{$\begin{array}{l}\text { Consumo } \\
\text { Intake }\end{array}$} & \multicolumn{3}{|c|}{$\begin{array}{c}\text { Nível de energia líquida } \\
\text { (Mcal/kg MS) } \\
\text { Level of net energy (Mcal/kg DM) }\end{array}$} \\
\hline & 1,1 & 1,4 & 1,6 \\
\hline $\begin{array}{l}\text { MS (g/dia) } \\
D M \text { (g/day) }\end{array}$ & $927,12^{b}$ & $1127,07^{\mathrm{a}}$ & $1094,21^{\mathrm{a}}$ \\
\hline $\begin{array}{l}\text { MS }\left(\mathrm{g} / \mathrm{kg}^{0,75}\right) \\
D M\left(\mathrm{~g} / \mathrm{kg}^{0.75}\right)\end{array}$ & $40,53^{b}$ & $48,93^{\mathrm{a}}$ & $48,48^{\mathrm{a}}$ \\
\hline $\begin{array}{l}\text { MS (\%PV) } \\
D M(\% B W)\end{array}$ & $1,43^{b}$ & $1,72^{\mathrm{a}}$ & $1,72^{\mathrm{a}}$ \\
\hline $\begin{array}{l}\text { FDN (g/dia) } \\
N D F \text { (g/day) }\end{array}$ & $579,07^{a}$ & $439,38^{b}$ & $250,15^{c}$ \\
\hline $\begin{array}{l}\text { FDN (\%PV) } \\
N D F(\% B W)\end{array}$ & $0,90^{\mathrm{a}}$ & $0,67^{\mathrm{b}}$ & $0,39^{\mathrm{c}}$ \\
\hline $\begin{array}{l}\text { EL (Mcal/dia) } \\
\text { NE (Mcal/day) }\end{array}$ & $1,06^{\mathrm{b}}$ & $1,60^{\mathrm{a}}$ & $1,78^{\mathrm{a}}$ \\
\hline $\begin{array}{l}\text { EL }\left(\mathrm{kcal} / \mathrm{kg}^{0,75}\right) \\
N E\left(\mathrm{~g} / \mathrm{kg}^{0.75}\right)\end{array}$ & $46,25^{c}$ & $69,69^{b}$ & $78,53^{\mathrm{a}}$ \\
\hline
\end{tabular}

Médias seguidas de uma mesma letra na linha não diferem pelo teste Student-Newman-Keuls a $5 \%$ de probabilidade.

Means in the same row with different superscripts differ by Student Newman Keuls test at $5 \%$ of probability.

MS e 64,98\% FDN estavam sujeitos à ação dos mecanismos físicos, pois a dieta continha pouca EL, enquanto aqueles que consumiram as dietas com 1,4 e 1,6 Mcal EL/kg MS 44,06 e 26,32\% de FDN, respectivamente, ficaram sujeitos aos mecanismos fisiológicos decorrentes dos níveis energéticos e de fibra destas dietas e apresentaram maiores consumos de MS.

Os valores máximos de consumo de FDN em relação aos pesos $(0,90 \% \mathrm{PV})$ observados nos animais consumindo dietas com alta concentração de fibra foram similares aos sugeridos por Mertens (1994) para vacas não-lactantes (0,89-0,91\%PV), o que comprova que o consumo de alimento é limitado em cabras no final da gestação consumindo dietas com grande quantidade de fibra ou volumoso, o que dificulta o atendimento de suas exigências nutricionais ou mesmo de correção de manejo inadequado que por acaso tenha ocorrido em fase anterior.

Nas recomendações do NRC (1981) para cabras gestantes, são considerados apenas partos simples, indicando acréscimo em torno de $20 \%$ para partos gemelares. Além disso, não existe recomendação considerando a necessidade de dividir o período seco em duas fases, tampouco considerações sobre o efeito da condição corporal das cabras no desempenho animal. Essas considerações também não foram observadas nas recomendações do AFRC (1997). Na última 


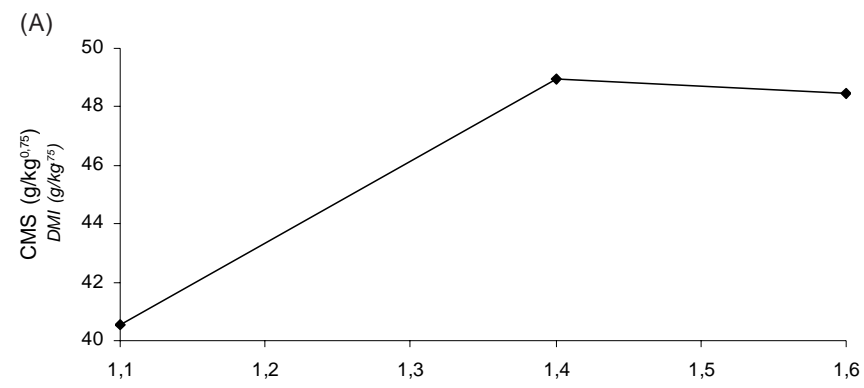

(B)

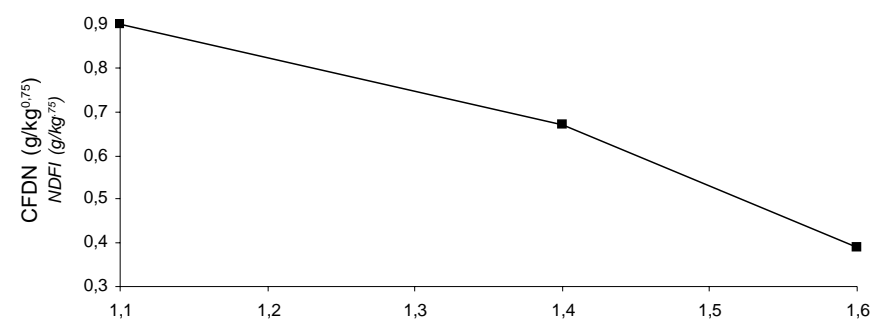

(C)

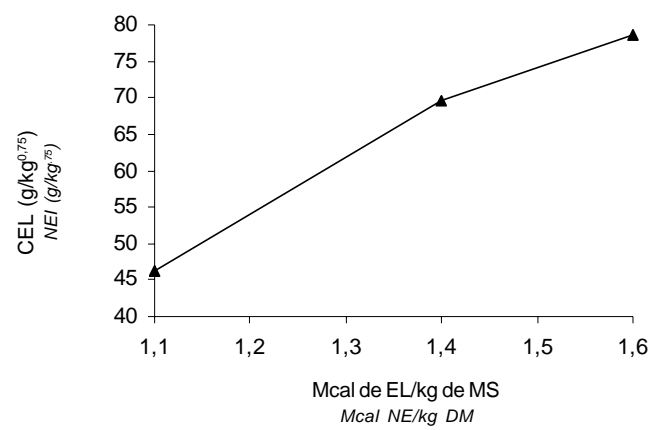

Figura 1 - Consumos de MS (CMS g/UTM) [A], FDN (CFDN \%PV) [B] e energia líquida (CEL kcal/UTM) [C] pelos animais alimentados com as dietas com níveis baixo $(1,1 \mathrm{Mcal}$ de EL/kg de MS), médio (1,4 Mcal de EL/kg de MS) e alto (1,6 Mcal de EL/kg de MS) de energia durante os últimos 30 dias de gestação.

Figure 1 - Intakes of DM (DMI g/kg $\left.{ }^{0.75}\right)[A], N D F(N D F I, \% B W)[B]$, and net energy (NEI kcal/UTM) [C] on goats fed diets with low (1.1 Mcal NE/kg DM), medium (1.4 Mcal NE/kg DM) or high (1.6 Mcal NE/kg DM) levels of net energy during the last 30 days of pregnancy.

edição publicada pela National Academy of Sciences dos Estados Unidos para bovinos leiteiros (NRC, 2001), esse tópico foi destacado em capítulo específico, como reconhecimento da importância do manejo alimentar no período de transição.

Outra consideração a ser discutida refere-se à possibilidade de partos múltiplos exercerem uma pressão maior na cavidade abdominal, provocando redução no volume ruminal. Essa possibilidade, embora não tenha sido avaliada neste experimento, constitui-se um fator a ser considerado. Essas observações reforçam a necessidade de se utilizar, como prática de manejo, duas dietas para o período seco, com os níveis mais elevados de forrageiras utilizados na primeira fase. Nos últimos 30 dias de gestação, seriam fornecidas dietas com maior concentração de energia visando reduzir as diferenças entre o consumo e o requerimento energético do animal.

Pesquisas como esta são realizadas com o objetivo de identificar um conteúdo ótimo de FDN na dieta que maximize o consumo de forrageiras e que, ao mesmo tempo, atenda aos requisitos de energia de um animal em determinado estado fisiológico. No entanto, ao comparar os consumos de EL ( $\mathrm{kcal} / \mathrm{kg}^{0,75}$ ) observados nos diferentes níveis de EL e os sugeridos pelo NRC (1981), de 127,63 kcal EL/UTM, para exigências na gestação, ou de 149,36 kcal EL/UTM, sugeridos por Moulin (1991) para cabras com $60 \mathrm{~kg}$, verificou-se redução no consumo de MS. Neste trabalho, o consumo de EL $\left(\mathrm{kcal} / \mathrm{kg}^{0,75}\right)$ diferiu entre os níveis energéticos das dietas $(\mathrm{P}<0,05)$, sendo menor no nível de 1,1 Mcal EL/kg MS (46,25 kcal EL/kg0,75) e maior no nível de 1,6 Mcal EL/kg MS (78,53 kcal/kg0,75) (Tabela 4). Essa constatação reforça a importância da utilização de dietas com densidade energética similar à daquelas utilizadas no início da lactação, durante os últimos 30 dias de gestação em caprinos (Figura 2).

A crescente mobilização de nutrientes neste período para atender à demanda pode ter implicações no parto e na lactação subseqüente. Apesar de não terem sido verificadas desordens metabólicas neste experimento, é provável que o impacto da redução de consumo tenha maiores implicações em situações nas quais os animais venham a apresentar gestações duplas ou triplas e com condição corporal que ultrapasse os limites sugeridos entre 2,5 e 4,0.

A análise de variância não mostrou efeito significativo $(\mathrm{P}>0,05)$ das interações condição corporal $\times$ nível de EL das dietas (Tabela 5). A condição corporal das cabras não influenciou o peso das crias, verifcando-se prolificidade média de 1,5 crias por cabra e peso médio das crias de

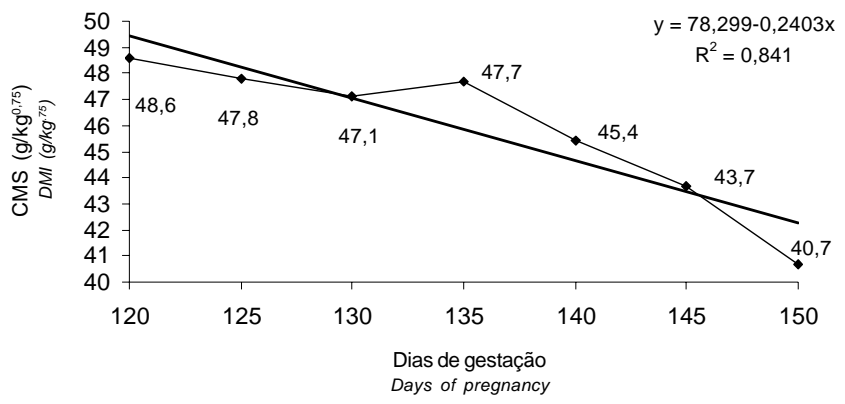

Figura 2 - Consumo médio de MS (CMS g/kg ${ }^{0,75}$ ) durante os últimos 30 dias de gestação.

Figure 2 - Average DM intake $\left(\mathrm{g} / \mathrm{kg}^{0.75}\right)$ during the last 30 days of pregnancy of dairy goats. 
Tabela 5 - Peso corporal de cabras com duas condições corporais alimentadas com três níveis de energia durante os últimos 30 dias de gestação

Table 5 - Body weight of goats with two different body condition score and receiving diets with three levels of net energy during the last 30 days of pregnancy

\begin{tabular}{|c|c|c|c|c|}
\hline \multirow{2}{*}{$\begin{array}{l}\text { Condição } \\
\text { corporal } \\
\text { Body condition } \\
\text { score }\end{array}$} & \multirow{2}{*}{$\begin{array}{c}\text { Nível de EL } \\
\text { (Mcal/kg MS) } \\
\text { Net energy } \\
\text { (Mcal/kg DM) }\end{array}$} & \multicolumn{3}{|c|}{$\begin{array}{l}\text { Dias para o parto } \\
\text { Days from birth }\end{array}$} \\
\hline & & -30 & -15 & $\begin{array}{l}\text { Parto } \\
\text { Birth }\end{array}$ \\
\hline $\begin{array}{l}\text { I } \\
(>3,25)\end{array}$ & $\begin{array}{l}1,1 \\
1,4 \\
1,6\end{array}$ & $\begin{array}{l}63,44 \\
61,95 \\
60,05\end{array}$ & $\begin{array}{l}64,11 \\
62,83 \\
60,14\end{array}$ & $\begin{array}{l}68,52 \\
65,80 \\
68,58\end{array}$ \\
\hline $\begin{array}{l}\text { II } \\
(<3,25)\end{array}$ & $\begin{array}{l}1,1 \\
1,4 \\
1,6\end{array}$ & $\begin{array}{l}63,56 \\
66,46 \\
69,45\end{array}$ & $\begin{array}{l}63,91 \\
67,21 \\
68,80\end{array}$ & $\begin{array}{l}66,35 \\
77,15 \\
67,85\end{array}$ \\
\hline
\end{tabular}

As médias seguidas de mesma letra na linha não diferem pelo teste Student Newman Keuls a $5 \%$ de probabilidade.

Means in the same row with different superscripts differ by Student Newman Keuls test at 5\% of probability.

3,68 kg. Ao contrário do observado neste experimento, Kayongo et al. (1984) verificaram que a suplementação préparto teve efeito no ganho de peso de ovelhas Red Marsai.

Analisando as curvas de concentração de AGNE no plasma de todos os animais durante as últimas três semanas de gestação (Figura 3), observou-se um comportamento não-linear, independentemente da condição corporal. Os valores de AGNE mantiveram-se relativamente constantes (média de 436, variando de 300 a 550 uM) entre os dias 21 a 7 antes do parto e apresentaram elevação significativa, com valores médios de 622 uM (variação de 500 a 800 uM) no dia do parto.

Apesar de os valores não terem sido estatisticamente diferentes, quando avaliado o efeito da condição corporal (Tabela 6), em geral, os maiores valores de AGNE no plasma foram obtidos nos animais com menor condição corporal $(<3,25)$. Este nível de AGNE pode estar relacionado ao maior consumo de MS pelos animais com pior condição corporal em comparação aos de melhor condição corporal (1.113,58 g/dia vs 985,36 g/dia). É provável que os maiores valores de AGNE no plasma dos animais de menor condição corporal tenham sido ocasionados pela utilização simultânea de mecanismos hormonais, permitindo maior mobilização de energia com o objetivo de reduzir o déficit energético. Entretanto, os níveis de EL nas dietas oferecidas influenciaram $(\mathrm{P}<0,05)$ a concentração de AGNE (mM) aos 14 dias antes da data prevista para o parto e no dia do parto e foram geralmente maiores para as dietas com menor nível de EL.

A observação sugere a presença de mecanismos de mobilização de energia (AGNE) que são rapidamente ativados no período final da gestação, o que reforça a

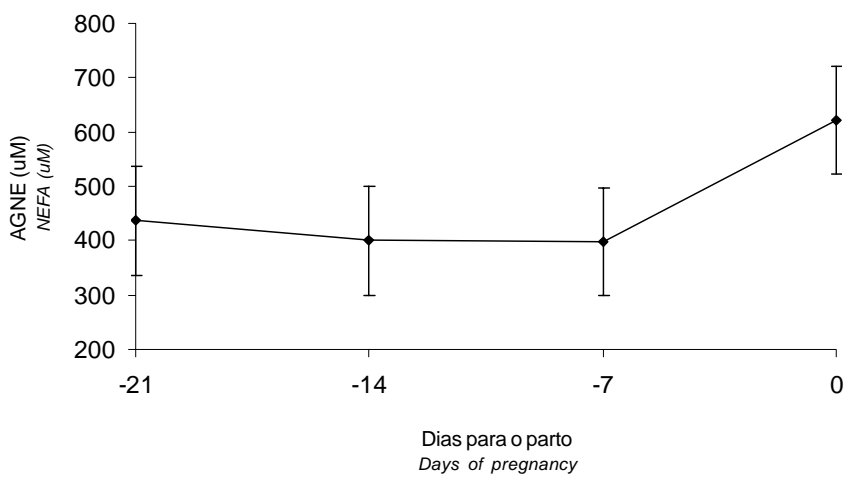

Figura 3 - Concentração média de ácidos graxos nãoesterificados (UM) durante os últimos 21 dias de gestação.

Figure 3 - Plasma concentration of nonesterified fatty acids (NEFA, UM) during the last 21 days of pregnancy.

Tabela 6 - Médias da concentração de AGNE (uM) no plasma de cabras em duas condições corporais alimentadas com dietas contendo três níveis de energia líquida durante os últimos 30 dias de gestação

Table 6 - Average NEFA content (UM) on goats with different body condition score and receiving diets with three levels of net energy during the last 30 days of pregnancy

Concentração de AGNE (uM) NEFA concentration $(u M)$

\begin{tabular}{|c|c|c|c|c|c|}
\hline \multirow[t]{2}{*}{$\begin{array}{l}\mathrm{CC} \\
B C S\end{array}$} & \multirow{2}{*}{$\begin{array}{c}\text { Energia líquida } \\
\text { (Mcal/kg MS) } \\
\text { Net energy } \\
\text { (Mcal/kg DM) }\end{array}$} & \multicolumn{4}{|c|}{$\begin{array}{l}\text { Dias para o parto } \\
\text { Days from birth }\end{array}$} \\
\hline & & -21 & -14 & -7 & 0 \\
\hline \multirow{3}{*}{$\begin{array}{l}\mathrm{I} \\
(>3,25)\end{array}$} & 1,1 & 714,34 & $653,42^{\mathrm{a}}$ & 551,71 & $651,67^{a}$ \\
\hline & 1,4 & 354,12 & $211,86^{b}$ & 187,38 & $522,30^{b}$ \\
\hline & 1,6 & 322,54 & $301,96^{\mathrm{b}}$ & 290,06 & $503,27^{b}$ \\
\hline \multirow{3}{*}{$\begin{array}{l}\text { II } \\
(<3,25)\end{array}$} & 1,1 & 397,62 & $472,13^{\mathrm{a}}$ & 468,38 & $958,68^{\mathrm{a}}$ \\
\hline & 1,4 & 556,02 & $367,18^{\mathrm{b}}$ & 502,48 & $596,40^{\mathrm{b}}$ \\
\hline & 1,6 & 274,56 & $391,63^{b}$ & 387,29 & $499,78^{b}$ \\
\hline
\end{tabular}

As médias seguidas de letra na linha diferem $(P<0,05)$ pelo teste Studen Newman Keuls.

Means in the same row with different superscripts differ $(P<0.05)$ by test Student Newman Keuls test.

importância de se reduzir a deficiência de energia nesse período, em especial para animais que apresentem partos múltiplos, em decorrência da alta demanda por nutrientes.

Segundo Reid et al. (1986), as concentrações plasmáticas de AGNE aumentam próximo ao parto, tanto em bovinos obesos (CC superior a 4,0) como em magros (CC inferior a 2,5).

Os valores das concentrações plasmáticas de AGNE, apesar de não terem sido estatisticamente diferentes nos dias 21 e 7 antes parto, comprovam uma tendência semelhante aos dados observados nos outros dias em que foram avaliados (Figura 4). O fornecimento da dieta com menor densidade energética promoveu maior mobilização de 
reservas corporais, o que reflete o menor consumo de energia observado nos animais daquele tratamento, aumentando o déficit entre oferta e demanda energética. Nesses animais, em todos os dias avaliados, os valores se mantiveram acima de $500 \mathrm{uM}$, o que reflete uma capacidade de resposta do animal durante o período de transição, quando fatores contribuem para a manifestação do déficit energético.

É provável que o aumento nas concentrações de AGNE nos últimos sete dias de gestação tenha tido uma contribuição mais acentuada das alterações hormonais que desencadeiam a liberação de AGNE no plasma que a própria dieta, visto que o percentual de aumento nos valores de AGNE entre os dias 7 e 0 foram aparentemente semelhantes e proporcionais aos níveis de cada tratamento.

Os níveis de AGNE não diferiram entre os animais alimentados com as dietas com 1,4 e 1,6 Mcal de EL/kg MS. Até o dia 7 antes ao parto, valores médios inferiores a 400 uM foram observados, o que pode ser considerado normal para a fase. Apesar do alto coeficiente de variação dos dados ( $\mathrm{CV}=30 \%)$, os valores de AGNE permitem sugerir essa variável como ferramenta de manejo nutricional no período de transição para auxiliar na identificação dos animais potencialmente sujeitos a desordens metabólicas.

Vazquez-Añon et al. (1994) afirmaram que o aumento de AGNE próximo ao parto e o pico no parto podem ser decorrentes da redução no consumo de matéria seca (MS) nesse período e das mudanças endócrinas. Antes do parto, as concentrações hormonais mudam para promover a gliconeogênese e a mobilização do tecido adiposo para prover de energia o feto e a glândula mamária. Assim, os hormônios lipolíticos próximo ao

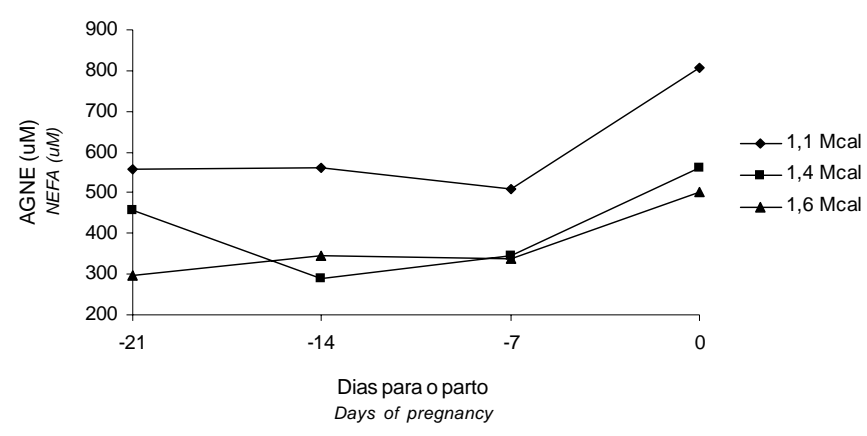

Figura 4 - Evolução da concentração de ácidos graxos nãoesterificados (UM) no sangue de animais com dietas com diferentes níveis de energia líquida durante os últimos 30 dias de gestação.

Figure 4 - Blood concentration of nonesterified fatty acids (NEFA, UM) on goats fed diets with three levels of net energy during the last 30 days of pregnancy. parto contribuem para o aumento de AGNE no plasma antes da redução no consumo de MS.

Outros autores relatam que as alterações endócrinas que sinalizam o processo de parto podem ser os fatores que aumentam a mobilização de gordura dos tecidos. O processo de mobilização de triglicerídeos do tecido adiposo tem início antes do parto. Nos últimos dias de gestação, o feto secreta cortisol (hormônio esteróide), aumentando os níveis desse hormônio e sinalizando o processo de parto (Goff \& Horst, 1997; Santos \& Santos, 1998).

Nas três últimas semanas de gestação, ocorre aumento na mobilização de triglicerídeos do tecido adiposo para suprir as necessidades dos animais no parto e na lactação, haja vista a redução no consumo de MS e as mudanças fisiológicas nesta fase (Grummer, 1995; Grum et al., 1996). Geralmente, o balanço de energia nesta fase é negativo e a demanda de nutrientes é maior que a ingestão, ocasionando aumento na taxa de lipólise e redução na taxa de lipogênese, promovendo a liberação de glicerol e AGNE (Chilliard, 1993; Santos \& Santos, 1998). Esses AGNE podem ser utilizados pelos tecidos como fonte de energia, precursores de gordura do leite, para a síntese de triglicerídeos e a geração de corpos cetônicos (Herdt \& Emery, 1992; Goff \& Horst, 1997). Durante o período de transição, as mudanças metabólicas devem ser coordenadas pelo estado fisiológico dos animais (gestação ou lactação) para suprir os nutrientes necessários durante o balanço energético negativo. As concentrações de AGNE são elevadas no período de 2 a 3 semanas pré-parto até o início da lactação, constituindo um indicativo do aumento da atividade lipolítica (Vernon, 1981; Riis, 1983) e da sensibilidade do tecido adiposo aos hormônios lipolíticos (ß- adrenérgicos).

\section{Conclusões}

Os níveis de energia líquida da dieta não ocasionaram variação significativa de peso nas cabras, pois a diferença entre os valores de condição corporal não foi suficiente para influenciar o desempenho dos animais.

A análise individual do efeito da condição corporal sobre o consumo mostrou que os animais com escore superior a 3,25 apresentaram menor consumo de MS e FDN. Provavelmente, as cabras mais gordas tiveram suas necessidades nutricionais supridas com menor quantidade de alimentos, por possuírem maior quantidade de reservas de energia, enquanto as mais magras consumiram mais alimento na tentativa de suprir as necessidades nutricionais dessa fase. Entretanto, a diferença entre os valores de condição corporal não foi suficiente para influenciar a concentração de AGNE no plasma dos animais. 
Os níveis de energia líquida das dietas influenciaram positivamente o consumo de MS e energia líquida durante o final da gestação. A dieta com 1,4 Mcal EL/kg MS foi mais indicada para cabras durante o final do período pré-parto, pois os animais apresentaram consumo de MS semelhante ao daqueles alimentados com a dieta mais energética.

O pico de AGNE durante o final da gestação ocorre quando o consumo de MS é reduzido, ou seja, nos últimos 21 dias antes do parto.

\section{Agradecimento}

Ao Departamento de Zootecnia da Universidade Federal de Viçosa, pela oportunidade e pelas condições de realizações deste trabalho.

À Coordenação de apoio à pesquisa (CAPES), pela concessão da bolsa de estudo.

Aos professores e funcionários do Departamento de Zootecnia, pelos ensinamentos e pela orientação na condução deste trabalho.

\section{Literatura Citada}

AGRICULTURAL AND FOOD RESEARCH COUNCIL - AFRC. The nutrition of goats. Nutrition Abstracts and Reviews (Series B), 1997. 830p.

CHILLIARD, Y. Dietary fat and adipose tissue metabolism in ruminants, pigs and rodents: a review. Journal of Dairy Science, v.76, n.12, p.3897-3931, 1993.

FORBES, J.M. Development of a model of voluntary food intake and energy balance in lactation cows. Animal Production, v.24, p.203-214, 1977.

FORBES, J.M. Voluntary feed intake. Quantitative aspects of ruminant digestion and metabolism. Cambridge: CAB International, University Press, 1993. p.479-494.

GOFF, J.P.; HORST, R.L. Physiological changes at parturition and their relationship to metabolic disorders. Journal of Dairy Science, v.80, n.7, p.1260-1268, 1997.

GRUM, D.E.; DRACKELY, J.K.; YOUNKER, R.S. et al. Nutrition during the dry period and hepatic lipid metabolism of periparturient dairy cows. Journal of Dairy Science, v.79, n.10, p.1850-1864, 1996.

GRUMMER, R. Impact of changes in organic nutrient metabolism on feeding the transition dairy cow. Journal of Animal Science, v.73, n.9, p.2820-2833, 1995.

HERDT, T.H.; EMERY, R.S. Therapy of diseases of ruminant intermediary metabolism. Veterinary Clinics of North America: Food Animal Pratice, v.8, p.91-106, 1992.

JOHNSON M.M.; PETERS, J.P. Technical note: an improved method to quantify nonesterified fatty acids in bovine plasma. Journal of Animal Science, v.71, n.3, p.753-756, 1993.

KAYONGO, S.B.; WANYOIKE, M.; KAYONGO-MALE, H. Red masai sheep: Effect of pre- and post-partum concentrate supplementation on performance. International Goat and Sheep Research, v.3, n.2, p.283-90, 1984.

MERTENS, D.R. Factors influencing feed intake in lactating cows: from theory to application using neutral detergent fiber. In: NUTRItion CONFERENCE, 1985, Georgia. Proceedings.. Georgia, 1985. p.1-18.

MERTENS, D.R. Predicting intake and digestibility using mathematical models of ruminal function. Journal of Animal Science v.64, p.1548-1558, 1987.

MERTENS, D.R. Regulation of feed intake. In: FAHEY JR., G.C. (Ed.). Forage quality, evaluation, and utilization. Madison: American Society of Agronomy, 1994. p.450-493.

MORAND-FEHR, P.; HERVIEU, J. Apprécier l'éat corporel des chèvres: Intérêt et méthod. Reussir La Chevre, n.231, p.2234, 1999.

MOULIN, C.H.S. Exigências energéticas para cabras em lactação. Viçosa, MG: Universidade Federal de Viçosa, 1991. 110p. Dissertação (Mestrado em Zootecnia) - Universidade Federal de Viçosa, 1991.

NATIONAL RESEARCH COUNCIL - NRC. Nutrient requirements of goats. Washington, D.C.: National Academy Science, 1981. 91p.

NATIONAL RESEARCH COUNCIL - NRC. Nutrient requirements of dairy cattle. 6.rev.ed. Washington, D.C.: National Academy Science, 1989. 158p.

NATIONAL RESEARCH COUNCIL - NRC. Nutrient requirements of dairy cattle. 7.rev.ed. Washington, D.C.: National Academy Science, 2001. 381p.

REID, I.M.; ROBERTS, C.J.; TREACHER, R.J. et al. Effect of body conditon at calving on tissue mobilization, development of fatty liver and blood chemistry of dairy cows. Animal Production, v.43, p.7-15, 1986.

RIIS, P.M. Adaptation of metabolism to various conditions: Nutritional and other environmental conditions. In: RIIS, P.M. (Ed.) Dynamic biochemistry of animal production. Amsterdan: World Animal Science, 1983. 501p.

SANTOS, J.E.P.; SANTOS, F.A.P. Novas estratégias no manejo e alimentação de vacas pré-parto. In: SIMPÓSIO SOBRE PRODUÇÃO ANIMAL, 10., 1998, Piracicaba. Anais... Piracicaba, 1998. p.165-214.

SILVA, D.J. Análise de alimentos (métodos químicos e biológicos). Viçosa, MG: Universidade Federal de Viçosa, 1990. $166 \mathrm{p}$.

STATISTICAL ANALISYS SYSTEM - SAS. SAS user's guide: statistics. version 6, 4.ed. Cary. North Carolina: 1990. 1686p.

Van SOEST, P.J.; ROBERTSON, J.B.; LEWIS, B.A. Methods of dietary fiber, neutral detergent fiber, and nonstarch polussaccharides in relation to animal nutrition. Journal of Dairy Science, v.74, p.3583-3597, 1991.

VAZQUEZ-AÑON, M.; BERTICS, S.; LUCK, M. et al. Peripartum liver triglyceride and plasma metabolites in dairy cows. Journal of Dairy Science, v.77, p.1521-1528, 1994.

VERNON, R.G. Lipid metabolism in the adipose tissue of ruminant animals. In: CHRISTIE, W.W. (Ed.). Lipid metabolism in ruminant animals. 1.ed. New York: Pergamon Press, 1981. p.279-362. 